\title{
Geometry of Submanifolds in Terms of Behavior of Geodesics
}

\author{
Sadahiro MAEDA and Koichi OGIUE
}

Shimane University and Tokyo Metropolitan University

\section{Introduction.}

It is interesting to study Riemannian submanifolds $M$ of a Riemannian manifold $\tilde{M}$ in terms of the behavior of geodesics of $M$. From this point of view, a totally geodesic submanifold is considered to be the simplest one. The second simplest one will be a circular geodesic submanifold, where every geodesic of $M$ is a circle in $\tilde{M}$. In the case where $\tilde{M}$ is a real space form, a circular geodesic submanifold has parallel second fundamental form.

We recall the notion of isotropic immersions introduced by O'Neill ([13]): Let $\sigma$ be the second fundamental form of $M$ in $\tilde{M}$. Then the immersion is said to be isotropic at $x \in M$ if $\|\sigma(X, X)\| /\|X\|^{2}$ is constant at $x$. If the immersion is isotropic at every point, then there exists a function $\lambda$ on $M$ defined by $x \rightarrow\|\sigma(X, X)\| /\|X\|^{2}$ and the immersion is said to be $\lambda$-isotropic or, simply, isotropic.

It is known that "circular geodesic" always implies "isotropic" and that the class of isotropic submanifolds is too wide to classify. The first author studied characterizations of circular geodesic submanifolds of a sphere among isotropic immersions. It is reasonable to study some class of submanifolds between "circular geodesic" and "isotropic".

The purpose of this paper is to characterize parallel immersions of a Cayley projective plane into a sphere among isotropic immersions and to show that complex hypersurfaces with parallel second fundamental form are characterized by a geometric condition weaker than "circular geodesic."

\section{Preliminaries.}

Let $(M, g)$ be an $n$-dimensional Riemannian submanifold of an $(n+p)$-dimensional Riemannian manifold $(\tilde{M}, \tilde{g})$. We denote by $\nabla$ and $\tilde{\nabla}$ the covariant differentiations on $M$ and $\tilde{M}$, respectively. Then the second fundamental form $\sigma$ of the immersion is defined 
by $\sigma(X, Y)=\tilde{\nabla}_{X} Y-\nabla_{X} Y$. We call $\mathfrak{h}=(1 / n)$ trance $\sigma$ the mean curvature vector of $M$ in $\tilde{M}$. The submanifold is said to be totally geodesic, totally umbilic or minimal provided that $\sigma=0, \sigma=g \otimes \mathfrak{h}$ or $\mathfrak{h}=0$, respectively. For a vector field $\xi$ normal to $M$, we put $\tilde{\nabla}_{X} \xi=-A_{\xi} X+D_{X} \xi$, where $-A_{\xi} X$ (resp. $D_{X} \xi$ ) denotes the tangential (resp. normal) component of $\tilde{\nabla}_{X} \xi$. We call $D$ the normal connection, and $\sigma$ and $A$ are related by $\tilde{g}(\sigma(X, Y), \xi)=g\left(A_{\xi} X, Y\right)$. A normal vector field $\xi$ is said to be parallel if $D \xi=0$. We define the covariant differentiation $\nabla^{\prime}$ of the second fundamental form $\sigma$ with respect to the connection in (tangent bundle) $\oplus$ (normal bundle) by

$$
\left(\nabla_{X}^{\prime} \sigma\right)(Y, Z)=D_{X}(\sigma(Y, Z))-\sigma\left(\nabla_{X} Y, Z\right)-\sigma\left(Y, \nabla_{X} Z\right) \text {. }
$$

The second fundamental form $\sigma$ is said to be parallel if $\nabla^{\prime} \sigma=0$.

We recall the Frenet formula for a curve $\gamma: I \rightarrow M$ parametrized by the arc length t. Let $e_{1}=\dot{\gamma}$ be the unit tangent vector and put $\kappa_{1}=\left\|\nabla_{j} e_{1}\right\|$ which is called the first curvature. If $\kappa_{1}=0$ on $I$, then $\gamma$ is said to be of order 1 . If $\kappa_{1}$ is not identically zero, then we define $e_{2}$ by $\nabla_{j} e_{1}=\kappa_{1} e_{2}$ on the set $I_{1}=\left\{t \in I \mid \kappa_{1}(t) \neq 0\right\}$. Put $\kappa_{2}=\left\|\nabla_{j} e_{2}+\kappa_{1} e_{1}\right\|$ which is called the second curvature. If $\kappa_{2}=0$ on $I_{1}$, then $\gamma$ is said to be of order 2 on $I_{1}$. If $\kappa_{2}$ is not identically zero on $I_{1}$, then we define $e_{3}$ by $\nabla_{j} e_{2}=-\kappa_{1} e_{1}+\kappa_{2} e_{3}$ on the set $I_{2}=\left\{t \in I_{1} \mid \kappa_{2}(t) \neq 0\right\}$. Inductively, we put $\kappa_{d}=\left\|\nabla_{j} e_{d}+\kappa_{d-1} e_{d-1}\right\|$ which is called the $d$-th curvature. If $\kappa_{d}=0$ on $I_{d-1}$, then $\gamma$ is said to be of order $d$ on $I_{d-1}$. If the curve $\gamma$ is of order $d$ on $I_{d-1}$, then we have a matrix equation

$$
\nabla_{\dot{\gamma}}\left(e_{1}, e_{2}, \cdots, e_{d}\right)=\left(e_{1}, e_{2}, \cdots, e_{d}\right) \Lambda,
$$

where $\Lambda$ is a $(d, d)$-matrix defined by

$$
\Lambda=\left(\begin{array}{cccc}
0 & -\kappa_{1} & & 0 \\
\kappa_{1} & 0 & \ddots & \\
& \ddots & \ddots & -\kappa_{d-1} \\
0 & & \kappa_{d-1} & 0
\end{array}\right)
$$

Equation (2.1) is called the Frenet formula. A curve $\gamma: I \rightarrow M$ is called a Frenet curve of $\operatorname{order} d(\leq \operatorname{dim} M)$ if there exist an orthonormal system $\left\{e_{1}=\dot{\gamma}, e_{2}, \cdots, e_{d}\right\}$ and positive functions $\left\{\kappa_{1}, \kappa_{2}, \cdots \kappa_{d-1}\right\}$ along $\gamma$ which satisfy a system of ordinary differential equation (2.1). The curve $\gamma$ is called a helix of order $d$ if all $\kappa_{i}^{\prime}$ 's are constant. In particular, a helix of order 2 is called a circle.

A submanifold $M$ of $\tilde{M}$ is said to be circular geodesic if every geodesic of $M$, considered as a curve in $\tilde{M}$, is a circle of $\tilde{M}$. The following result clarifies the fundamental property of circular geodesic submanifolds.

Proposition A ([10]). A submanifold $M$ of $\tilde{M}$ is circular geodesic if and only if $M$ is nonzero isotropic and the second fundamental form $\sigma$ satisfies $\left(\nabla^{\prime}{ }_{X} \sigma\right)(X, X)=0$ for all $X$ tangent to $M$.

As a consequence of Proposition A, we have the following. 
REMARK 1. (1) "Circular geodesic" implies "isotropic".

(2) If $M$ is circular geodesic in $\tilde{M}$, then every geodesic of $M$, considered as a curve in $\tilde{M}$, has the same first curvature.

(3) In the case where $\tilde{M}$ is a real space form, "circular geodesic" implies $\nabla^{\prime} \sigma=0$.

We recall the following.

LEMMA 1 ([2]). Let $M$ be an n-dimensional minimal submanifold of an $(n+p)$ dimensional real space form $\tilde{M}(c)$ of constant curvature $c$. Then the second fundamental form satisfies

$$
\begin{aligned}
\frac{1}{2} \Delta\|\sigma\|^{2}= & \left\|\nabla^{\prime} \sigma\right\|^{2}-2 \sum\left(h_{i j}^{\alpha} h_{j k}^{\alpha} h_{k l}^{\beta} h_{l i}^{\beta}-h_{i j}^{\alpha} h_{j k}^{\beta} h_{k l}^{\alpha} h_{l i}^{\beta}\right) \\
& -\sum h_{i j}^{\alpha} h_{i j}^{\beta} h_{k l}^{\alpha} h_{k l}^{\beta}+n c\|\sigma\|^{2},
\end{aligned}
$$

where $h_{i j}^{\alpha}$ denote the components of $\sigma$ with respect to a local orthonormal frame fields and $\Delta$ denotes the Laplacian.

We denote by $K(X, Y)$ (resp. $\tilde{K}(X, Y))$ the sectional curvature of $M$ (resp. $\tilde{M})$ given by the plane spanned by $X$ and $Y$ and put $\Delta_{X Y}=K(X, Y)-\tilde{K}(X, Y)$. We call $\Delta$ the discriminant. The following is due to O'Neill.

LeMMA 2 ([13]). Let $M^{n}$ be a $\lambda(>0)$-isotropic submanifold in a Riemannian manifold $\tilde{M}^{n+p}$. Assume that the discriminant $\Delta$ is constant on a $k$-dimensional linear subspace $V$ of $T_{x}(M)$. Then $-\left((k+2) /(2(k-1)) \lambda^{2} \leq \Delta \leq \lambda^{2}\right.$ on $V$. Moreover, $\Delta=\lambda^{2}$ on $V$ if $\operatorname{dim}\{\sigma(X, Y) \mid X, Y \in V\}_{\boldsymbol{R}}<(k(k+1)) / 2-1$.

Let $M$ be a submanifold in a real space form $\tilde{M}(c)$ of constant curvature $c$. Then the equations of Gauss and Codazzi are given respectively by

$$
\begin{aligned}
g(R(X, Y) Z, W)= & c\{g(Y, Z) g(X, W)-g(X, Z) g(Y, W)\} \\
& +g(\sigma(Y, Z), \sigma(X, W))-g(\sigma(X, Z), \sigma(Y, W))
\end{aligned}
$$

and

$$
\left(\nabla^{\prime}{ }_{X} \sigma\right)(Y, Z)=\left(\nabla^{\prime}{ }_{Y}\right)(X, Z),
$$

where $R$ denotes the curvature tensor of $M$.

We also prepare the following.

LEMMA 3 ([6]). Let $M$ be a constant isotropic submanifold in a real space form and assume that $M$ is locally symmetric and the first normal space coincides with the normal space at each point of $M$. Then the second fundamental form of the immersion is parallel.

We denote by Cay the set of Cayley numbers, which is an 8-dimensional non-associative division algebra over the real numbers. It has multiplicative identity and a positive definite bilinear form $\langle$,$\rangle . Let M$ be either the Cayley projective 
plane or its noncompact dual. Then the tangent space of $M$ may be identified with Cay $\oplus$ Cay, which is considered as the set of ordered pairs of Cayley numbers. The vector space $C a y \oplus C a y$ has a positive definite symmetric bilinear form $\langle$,$\rangle given$ by $\langle(a, c),(b, d)\rangle=\langle a, b\rangle+\langle c, d\rangle$ (for detail, see [1]). The curvature tensor $R$ of $M$ is given by

$$
\begin{aligned}
& \langle R((a, b),(c, d))(e, f),(g, h)\rangle \\
= & \alpha(\langle c, e\rangle\langle a, g\rangle-\langle a, e\rangle\langle c, g\rangle+\langle d, f\rangle\langle b, h\rangle-\langle b, f\rangle\langle d, h\rangle) \\
& +\frac{\alpha}{4}(\langle e d, g b\rangle-\langle e b, g d\rangle+\langle c f, a h\rangle-\langle a f, c h\rangle+\langle a d-c b, g f-e h\rangle),
\end{aligned}
$$

where $\alpha$ is a nonzero real number.

\section{Results.}

As for submanifolds of a real space form, the class of circular geodesic submanifolds is completely determined because they have parallel second fundamental forms, whereas the class of isotropic submanifolds seems to be too wide to classify. Therefore it is reasonable to study isotropic submanifolds of a real space form under some additional condition. We first prove the following.

THEOREM 1. Let $M$ be a connected open submanifold of either the Cayley projective plane or its noncompact dual and $\tilde{M}^{16+p}(c)$ be a real space form of curvature c. If $p \leq 10$ and $M$ admits an isometric immersion as an isotropic submanifold of $\tilde{M}^{16+p}(c)$, then $p=9$ or 10 and

(i) $M$ is locally congruent to a connected open submanifold of the Cayley projective plane which is immersed into $\tilde{M}^{25}(c)$ through the first standard minimal immersion, or

(ii) $M$ is locally congruent to a connected open submanifold of the Cayley projective plane which is immersed into some totally umbilical hypersurface of $\tilde{M}^{26}(c)$ through the first standard minimal immersion.

Proof. We immediately find from (2.5) that

$$
K((a, 0),(b, 0))=\langle R((a, 0),(b, 0))(b, 0),(a, 0)\rangle=\alpha \quad \text { if } \quad(a, 0) \wedge(b, 0) \neq 0 .
$$

This implies that the discriminant $\Delta$ restricted to $C a y \oplus\{0\}$ is constant at each point. Assume that $M$ is $\lambda$-isotropic in $\tilde{M}^{16+p}(c)$. Then Lemma 2 asserts that

$$
\lambda^{2}=\alpha-c
$$

since $p<35=8 \times 9 / 2-1$.

On the other hand, let $\sigma$ be the second fundamental form of $M$ in $\tilde{M}^{16+p}(c)$. Then we have $\|\sigma(X, X)\|=\lambda\|X\|^{2}$ for all $X$, which is equivalent to 


$$
\begin{array}{r}
g(\sigma(X, Y), \sigma(Z, W))+g(\sigma(X, Z), \sigma(Y, W))+g(\sigma(X, W), \sigma(Y, Z)) \\
=\lambda^{2}\{g(X, Y) g(Z, W)+g(X, Z) g(Y, W)+g(X, W) g(Y, Z)\}
\end{array}
$$

for all $X, Y, Z$ and $W$. From (2.3) and (3.1), we obtain

$$
\begin{aligned}
3 g(\sigma & (X, Y), \sigma(Z, W))=g(R(Z, X) Y, W)+g(R(Z, Y) X, W) \\
& -c\{2 g(X, Y) g(Z, W)-g(Y, Z) g(X, W)-g(X, Z) g(Y, W)\} \\
& +\lambda^{2}\{g(X, Y) g(Z, W)+g(X, Z) g(Y, W)+g(Y, Z) g(X, W)\} .
\end{aligned}
$$

We see from (2.5), (3.1) and (3.3) that

$$
\begin{aligned}
& \langle\sigma((a, b),(c, d)), \sigma((e, f),(g, h))\rangle \\
& =(\alpha-c)(\langle a, c\rangle\langle e, g\rangle+\langle b, d\rangle\langle f, h\rangle) \\
& \quad-c(\langle b, d\rangle\langle e, g\rangle+\langle a, c\rangle\langle f, h\rangle) \\
& +\frac{\alpha}{3}\{\langle b, d\rangle\langle e, g\rangle+\langle a, c\rangle\langle f, h\rangle+\langle a, g\rangle\langle d, f\rangle \\
& \quad+\langle b, h\rangle\langle c, e\rangle+\langle b, f\rangle\langle c, g\rangle+\langle a, e\rangle\langle d, h\rangle\} \\
& +\frac{\alpha}{12}\{\langle c b, e h\rangle+\langle a h, c f\rangle+\langle a d, g f\rangle+\langle e d, g b\rangle \\
& \quad+\langle c b, g f\rangle+\langle a f, c h\rangle+\langle a d, e h\rangle+\langle e b, g d\rangle \\
& \quad-2\langle c h, e b\rangle-2\langle a f, g d\rangle-2\langle c f, g b\rangle-2\langle a h, e d\rangle\}
\end{aligned}
$$

For simplicity, we put $X_{i}=\left(e_{i}, 0\right)$ and $Y_{i}=\left(0, e_{i}\right)$ for $0 \leq i \leq 7$, where $\left\{e_{0}=1, e_{1}, \cdots, e_{7}\right\}$ is a basis for Cay. By using (3.4), we see that the vectors $\sigma\left(X_{0}, X_{0}\right), \sigma\left(X_{0}, Y_{0}\right)$, $\sigma\left(X_{0}, Y_{1}\right), \cdots, \sigma\left(X_{0}, Y_{7}\right)$ are nonzero and mutually orthogonal. Let $v_{x}^{1}$ denote the first normal space of $M$ at $x$. Then we have $\operatorname{dim} v_{x}^{1} \geq 9$ for all $x \in M$. We shall prove in the following that the second fundamental form $\sigma$ is parallel.

Case (I): $p \leq 9$. Note that the first normal space coincides with the normal space at each point of $M$. This, combined with the fact that $\lambda$ is constant, implies that the second fundamental form of the immersion is parallel (cf. Lemma 3). Therefore, due to the work of Ferus ([3]), we see that $M$ is locally congruent to a connected open submanifold of the Cayley projective plane and the immersion is locally equivalent to the first standard minimal immersion.

Case (II): $p=10$. Our discussion is divided into the following two subcases:

Case (IIa): $\sigma\left(X_{0}, X_{0}\right)$ and $\sigma\left(Y_{0}, Y_{0}\right)$ are linearly independent. We see from (3.4) that $\sigma\left(Y_{0}, Y_{0}\right), \sigma\left(X_{0}, Y_{0}\right), \sigma\left(X_{0}, Y_{1}\right), \cdots, \sigma\left(X_{0}, Y_{7}\right)$ are mutually orthogonal nonzero vectors so that $\operatorname{dim} v_{x}^{1} \geq 10$ for each $x \in M$. Therefore the same argument as in Case (I) asserts that the manifold $M$ is a parallel submanifold. Hence, by virtue of the work of Ferus ([3]), we find that $M$ is locally congruent to a connected open submanifold of the Cayley projective plane which is immersed into some totally umbilical (not totally 
geodesic) hypersurface of $\tilde{M}^{26}(c)$ through the first standard minimal immersion.

Case (IIb): $\sigma\left(X_{0}, X_{0}\right)$ and $\sigma\left(Y_{0}, Y_{0}\right)$ are linearly dependent. We have

$$
\left|\left\langle\sigma\left(X_{0}, X_{0}\right), \sigma\left(Y_{0}, Y_{0}\right)\right\rangle\right|=\left\|\sigma\left(X_{0}, X_{0}\right)\right\|\left\|\sigma\left(Y_{0}, Y_{0}\right)\right\|
$$

which, together with (3.4), implies that $|\alpha / 2-c|=|\alpha-c|$ so that $\alpha=(4 / 3) c$. Substituting $\alpha=(4 / 3)$ c into (3.4), we get an equation, say, (3.4)', which implies that our immersion is minimal. On the other hand, the first standard minimal immersion $f$ of the Cayley projective plane of maximal sectional curvature $(4 / 3) c$ into a 25-dimensional Euclidean sphere of curvature $c$ is a parallel immersion. Moreover, the immersion $f$ also satisfies (3.4)'. This, together with Lemma 1 , implies that the immersion is a parallel immersion. Hence we can see that $M$ is locally congruent to a connected open submanifold of the Cayley projective plane which is immersed into some totally geodesic hypersurface of $\tilde{M}^{26}(c)$ through the first standard minimal immersion (cf. [3]).

REMARK 2. Let $f: P_{\text {Cay }}^{2}((4 / 3) c) \rightarrow S^{25}(c)$ be the first standard minimal immersion of the Cayley projective plane (of maximal sectional curvature $(4 / 3) c$ ) into a 25-dimensional sphere (of curvature c). We see from (3.4)' that $\sqrt{(3 / c)} \sigma\left(X_{0}, X_{0}\right), \sqrt{(3 / c)} \sigma\left(X_{0}, Y_{0}\right)$, $\sqrt{(3 / c)} \sigma\left(X_{0}, Y_{1}\right), \cdots, \sqrt{(3 / c)} \sigma\left(X_{0}, Y_{7}\right)$ form an orthonormal basis for the first normal space of $f$ and that

$$
\begin{aligned}
& \sigma\left(X_{0}, X_{0}\right)=\sigma\left(X_{i}, X_{i}\right)=-\sigma\left(Y_{i}, Y_{i}\right) \quad \text { for } 0 \leq i \leq 7 \\
& \sigma\left(X_{i}, X_{j}\right)=\sigma\left(Y_{i}, Y_{j}\right)=0 \quad \text { for } 0 \leq i \neq j \leq 7 \\
& \sigma\left(X_{0}, Y_{0}\right)=-\sigma\left(X_{i}, Y_{i}\right) \quad \text { for } \quad 1 \leq i \leq 7 \\
& \sigma\left(X_{0}, Y_{1}\right)=\sigma\left(X_{2}, Y_{3}\right)=\sigma\left(X_{4}, Y_{5}\right)=-\sigma\left(X_{6}, Y_{7}\right) \\
& \sigma\left(X_{0}, Y_{2}\right)=-\sigma\left(X_{1}, Y_{3}\right)=\sigma\left(X_{4}, Y_{6}\right)=\sigma\left(X_{5}, Y_{7}\right) \\
& \sigma\left(X_{0}, Y_{3}\right)=\sigma\left(X_{1}, Y_{2}\right)=\sigma\left(X_{4}, Y_{7}\right)=-\sigma\left(X_{5}, Y_{6}\right) \\
& \sigma\left(X_{0}, Y_{4}\right)=-\sigma\left(X_{1}, Y_{5}\right)=-\sigma\left(X_{2}, Y_{6}\right)=-\sigma\left(X_{3}, Y_{7}\right) \\
& \sigma\left(X_{0}, Y_{5}\right)=\sigma\left(X_{1}, Y_{4}\right)=-\sigma\left(X_{2}, Y_{7}\right)=\sigma\left(X_{3}, Y_{6}\right) \\
& \sigma\left(X_{0}, Y_{6}\right)=\sigma\left(X_{1}, Y_{7}\right)=\sigma\left(X_{2}, Y_{4}\right)=-\sigma\left(X_{3}, Y_{5}\right) \\
& \sigma\left(X_{0}, Y_{7}\right)=-\sigma\left(X_{1}, Y_{6}\right)=\sigma\left(X_{2}, Y_{5}\right)=\sigma\left(X_{3}, Y_{4}\right) .
\end{aligned}
$$

The first author proved the following characterizations of circular geodesic submanifolds.

THEOREM A ([5], [9]). Let $M(c)$ be an n-dimensional real space form of constant curvature $c$ isotropically immersed in an $(n+p)$-dimensional real space form $\tilde{M}(\tilde{c})$ of constant curvature $\tilde{c}$. If $c<\tilde{c}$ and $p \leq \frac{1}{2}\left(n^{2}+n-2\right)$, then $p=\frac{1}{2}\left(n^{2}+n-2\right)$ and $M(c)$ is immersed as a Veronese manifold in $\tilde{M}(\tilde{c})$.

THEOREM B ([7]). Let $M$ be an $n$-dimensional complex space form $(n \geq 2)$ isotropically immersed in $a(2 n+p)$-dimensional real space form $\tilde{M}(c)$ of constant curvature 
$c>0$. If $p \leq n^{2}$, then $p=n^{2}-1$ or $p=n^{2}$ and $M$ is one of the following:

(i) $M$ is locally congruent to a complex projective space which is immersed into $\tilde{M}(c)$ through the first standard minimal immersion.

(ii) $M$ is locally congruent to a complex projective space which is immersed into some totally umbilical hypersurface of $\tilde{M}(c)$ through the first standard minimal immersion.

THEOREM C ([8]). Let $M$ be an n-dimensional quaternionic space form isotropically immersed in a $(4 n+p)$-dimensional real space form $\tilde{M}(c)$ of constant curvature $c>0$. If $p<2 n^{2}+2 n-1$, then $p=2 n^{2}-n-1$ or $p=2 n^{2}-n$ and $M$ is one of the following:

(i) $M$ is locally congruent to a quaternionic projective space which is immersed into $\tilde{M}(c)$ through the first standard minimal immersion.

(ii) $M$ is locally congruent to a quaternionic projective space which is immersed into some totally umbilical hypersurface of $\tilde{M}(c)$ through the first standard minimal immersion.

We next consider submanifolds of a complex space form. The class of circular geodesic submanifolds of a complex space form has not yet been determined. We will deal with complex hypersurfaces in a complex space form satisfying a condition weaker than "circular geodesic", which can be considered as a generalization of a result of Nomizu ([12]).

THEOREM 2. Let $M$ be a complex hypersurface of a complex space form $\tilde{M}(c)$. Then every geodesic of $M$, considered as a curve in $\tilde{M}(c)$, has constant first curvature if and only if $M$ is totally geodesic or locally congruent to a complex quadric, the latter case arising only when $c>0$.

Proof. Let $\gamma=\gamma(t)$ be a geodesic of $M$. Then we see that $\|\sigma(\dot{\gamma}, \dot{\gamma})\|$ is constant along $\gamma$. This implies that $g\left(\left(\nabla_{\dot{\gamma}}^{\prime} \sigma\right)(\dot{\gamma}, \dot{\gamma}), \sigma(\dot{\gamma}, \dot{\gamma})\right)=0$ and hence we have

$$
g\left(\left(\nabla_{X}^{\prime} \sigma\right)(X, X), \sigma(X, X)\right)=0
$$

for every vector field $X$ tangent to $M$. Let $J$ be the complex structure. Then, by replacing $X$ by $J X$, we obtain

$$
g\left(\left(\nabla_{X}^{\prime} \sigma\right)(X, X), J \sigma(X, X)\right)=0
$$

for all $X$. It follws from (3.5) and (3.6) that $\left(\nabla_{X}^{\prime} \sigma\right)(X, X)=0$ for all $X$. This implies that the second fundamental form $\sigma$ is parallel. Thus we get the conclusion (cf. [11]).

Theorem 2 can also be considered as a complex analogue of the following: Let $M$ be a hypersurface of a real space form. Then every geodesic of $M$, considered as a curve in the ambient space, has constant first curvature if and only if' $M$ has parallel second fundamental form. 


\section{References}

[1] R. B. Brown and A. GraY, Riemannian manifolds with holonomy group Spin(9), Differential Geometry, in honor of K. Yano, Kinokuniya (1972), 41-59.

[2] S. S. Chern, M. P. DoCarmo and S. Kobayashi, Minimal submanifolds of a sphere with second fundamental form of constant length, Functional Analysis and Related Fields, Springer (1970), 59-75.

[ 3 ] D. Ferus, Immersions with parallel second fundamental form, Math. Z. 140 (1974), 87-93.

[4] S. Ishihara, Quaternion Kaehlerian manifolds, J. Diff. Geom. 9 (1974), 483-500.

[ 5 ] S. MAEDA, Isotropic immersions with parallel second fundamental form, Canad. Math. Bull. 26 (1983), 291-296.

[6] S. MAEDA, Isotropic immersions with parallel second fundamental form II, Yokohama Math. J. 31 (1983), 131-138.

[ 7 ] S. MAEDA, Isotropic immersions, Canad. J. Math. 38 (1986), 416-430.

[ 8 ] S. MAEDA, Remarks on isotropic immersions, Yokohama Math. J. 34 (1986), 83-90.

[9] S. MAEDA, Differential geometry of constant mean curvature submanifolds, Mem. Fac. Gen. Ed. Kumamoto Univ. 24 (1989), 7-39.

[10] S. MAEDa and N. Sato, On submanifolds all of whose geodesics are circles in a complex space form, Kodai Math. J. 6 (1983), 157-166.

[11] H. NaKagawa and R. TAKagi, On locally symmetric Kaehler submanifolds in a complex projective space, J. Math. Soc. Japan 28 (1976), 638-667.

[12] K. Nomizu, A characterization of the Veronese varieties, Nagoya Math. J. 60 (1976), 181-186.

[13] B. O’NelLL, Isotropic and Kaehler immersions, Canad. J. Math. 17 (1965), 905-915.

Present Addresses:

SADAHIRO MaEda

Department of Mathematics, Shimane University, MATSUE, 690 JAPAN.

KoICHI OGIUE

Department of Mathematics, Tokyo Metropolitan University, HACHIOJ, TOKYO, 192-03 JAPAN. 